\title{
A uniform realization of the combinatorial $R$-matrix
}

\author{
Cristian Lenart and Arthur Lubovsky
}

Department of Mathematics and Statistics, State University of New York at Albany, Albany, NY 12222, USA

\begin{abstract}
Kirillov-Reshetikhin (KR) crystals are colored directed graphs encoding the structure of certain finitedimensional representations of affine Lie algebras. A tensor product of column shape KR crystals has recently been realized in a uniform way, for all untwisted affine types, in terms of the quantum alcove model. We enhance this model by using it to give a uniform realization of the combinatorial $R$-matrix, i.e., the unique affine crystal isomorphism permuting factors in a tensor product of KR crystals. In other words, we are generalizing to all Lie types Schützenberger's sliding game (jeu de taquin) for Young tableaux, which realizes the combinatorial $R$-matrix in type $A$. We also show that the quantum alcove model does not depend on the choice of a sequence of alcoves.

Résumé. Les cristaux de Kirillov-Reshetikhin (KR) sont des graphes orientés avec des arêtes étiquetées qui encodent certaines représentations de dimension finie des algèbres de Lie affines. Les produits tensoriels des cristaux KR de type colonne ont été récemment réalisés de manière uniforme, pour tous les types affines symétriques, en termes du modèle des alcôves quantique. Nous enrichons ce modèle en l'utilisant pour donner une réalisation uniforme de la $R$-matrice combinatoire, c'est à dire, l'isomorphisme des cristaux affines unique quit permute les facteurs dans un produit tensoriel des cristaux KR. En d'autres termes, nous généralisons pour tous les types de Lie le jeu de taquin de Schützenberger sur les tableaux de Young, qui réalise la $R$-matrice combinatoire dans le type $A$. Nous montrons aussi que le modèle des alcôves quantique ne dépend pas du choix d'une suite d'alcôves.
\end{abstract}

Keywords: Kirillov-Reshetikhin crystals, energy function, combinatorial $R$-matrix, quantum alcove model.

\section{Introduction}

Kashiwara's crystals [Kas91] are colored directed graphs encoding the structure of certain bases (called crystal bases) of some representations of quantum groups $U_{q}(\mathfrak{g})$ as $q$ goes to zero (where $\mathfrak{g}$ is a symmetrizable Kac-Moody Lie algebra). All highest weight representations have crystal bases/graphs. Beside them, an important class of crystals is represented by the Kirillov-Reshetikhin (KR) crystals [KR90]. They correspond to certain finite-dimensional modules for affine Lie algebras which are not of highest weight. A $\mathrm{KR}$ crystal is denoted $B^{r, s}$, being labeled by an $r \times s$ rectangle, where the height $r$ indexes a simple root of the corresponding finite root system and the width $s$ is any positive integer. The importance of KR

\footnotetext{
${ }^{\dagger}$ Partially supported by the NSF grants DMS-1101264 and DMS-1362627; the hospitality and support of the Max-PlanckInstitut für Mathematik in Bonn, where part of this work was carried out, are also gratefully acknowledged.

${ }^{\ddagger}$ Supported by a GAANN grant from the US Department of Education.

1365-8050 @ 2015 Discrete Mathematics and Theoretical Computer Science (DMTCS), Nancy, France
} 
crystals stems from the fact that they are building blocks for the corresponding (infinite) highest weight crystals; indeed, the latter are realized as infinite tensor products of the former in the Kyoto path model, see, e.g., [HK00]. Tensor products of KR crystals are endowed with a grading known as the energy function, which originates in the theory of solvable lattice models [ $\left.\mathrm{HKO}^{+} 99\right]$. There is a unique affine crystal isomorphism between two tensor products of KR crystals differing by a permutation of the tensor factors; it is called the combinatorial R-matrix.

The first author and Postnikov [LP07, LP08] defined the so-called alcove model for highest weight crystals associated to a symmetrizable Kac-Moody algebra. This model is a discrete counterpart of the celebrated Littelmann path model [Lit95]. In [LL14] the authors generalize the alcove model. This generalization, called the quantum alcove model, has been shown in $\left[\mathrm{LNS}^{+} 13 \mathrm{~b}\right]$ to uniformly describe tensor products of column shape KR crystals for all untwisted affine types. By contrast, all the existing combinatorial models for KR crystals are type-specific; most of them correspond to the classical types, and are based on diagram fillings, i.e., on tableau models [FOS09]. As far as the energy function is concerned, in the quantum alcove model it is computed uniformly and efficiently by a statistic called height [ $\left.\mathrm{LNS}^{+} 13 \mathrm{~b}\right]$, whereas an efficient computation based on the tableau models is only available in types $A$ and $C$ [Len12, LS13].

In this paper we enhance the quantum alcove model by using it to give a uniform realization of the combinatorial $R$-matrix. The construction is based on certain combinatorial moves called quantum YangBaxter moves, which generalize their alcove model versions defined in [Len07]. Note that, as far as existing realizations of the combinatorial $R$-matrix are concerned, they are limited in scope and type-specific. For instance, in terms of the tableau models, there is a construction in type $A$ based on Schützenberger's jeu de taquin (sliding algorithm) on two columns [Ful97], whereas the extensions of this procedure to types $B$ and $C$ are involved and not transparent, see [Lec02, Lec03]. By contrast, our construction is easy to formulate, and is related to more general concepts.

We also show that, like the alcove model, its quantum generalization does not depend on the choice of a sequence of roots called a $\lambda$-chain (or, equivalently, on the choice of a sequence of alcoves joining the fundamental one to a translation of it). Note that the similar statement for the Littelmann path model was proved in [Lit95] based on subtle continuous arguments, whereas the alcove model and its quantum generalization are discrete, so they are amenable to the use of the mentioned combinatorial methods. Due to space constraints we were unable to include any proofs in this extended abstract and instead refer the interested reader to [LL15].

\section{Background}

\subsection{Root systems}

Let $\mathfrak{g}$ be a complex simple Lie algebra, and $\mathfrak{h}$ a Cartan subalgebra, of rank $r$. Let $\Phi \subset \mathfrak{h}^{*}$ be the corresponding irreducible root system, $\mathfrak{h}_{\mathbb{R}}^{*} \subset \mathfrak{h}^{*}$ the real span of the roots, and $\Phi^{+} \subset \Phi$ the set of positive roots. We denote, as usual, the reflection corresponding to the root $\alpha$ by $s_{\alpha}$. Let $\alpha_{1}, \ldots, \alpha_{r} \in \Phi^{+}$be the simple roots, and $s_{i}:=s_{\alpha_{i}}$ the simple reflections; the latter generate the Weyl group $W$. We denote $\langle\cdot, \cdot\rangle$ the non-degenerate scalar product on $\mathfrak{h}_{\mathbb{R}}^{*}$ induced by the Killing form. Given a root $\alpha$, the corresponding coroot is $\alpha^{\vee}:=2 \alpha /\langle\alpha, \alpha\rangle$. If $\alpha=\sum_{i} c_{i} \alpha_{i}$, then the height of $\alpha$ is given by ht $(\alpha):=\sum_{i} c_{i}$.

The weight lattice $\Lambda$ consists of $\lambda \in \mathfrak{h}_{\mathbb{R}}^{*}$ with $\left\langle\lambda, \alpha^{\vee}\right\rangle \in \mathbb{Z}$ for any $\alpha \in \Phi$. The weight lattice $\Lambda$ is generated by the fundamental weights $\omega_{1}, \ldots \omega_{r}$, which form the dual basis to the basis of simple coroots, i.e., $\left\langle\omega_{i}, \alpha_{j}^{\vee}\right\rangle=\delta_{i j}$. The set $\Lambda^{+}$of dominant weights consists of $\lambda \in \Lambda$ with $\left\langle\lambda, \alpha^{\vee}\right\rangle \geq 0$ for any $\alpha \in \Phi^{+}$. 
Given $\alpha \in \Phi$ and $k \in \mathbb{Z}$, we denote by $s_{\alpha, k}$ the reflection in the affine hyperplane

$$
H_{\alpha, k}:=\left\{\lambda \in \mathfrak{h}_{\mathbb{R}}^{*}:\left\langle\lambda, \alpha^{\vee}\right\rangle=k\right\} .
$$

These reflections generate the affine Weyl group $W_{\text {aff }}$ for the dual root system $\Phi^{\vee}:=\left\{\alpha^{\vee} \mid \alpha \in \Phi\right\}$. The hyperplanes $H_{\alpha, k}$ divide the real vector space $\mathfrak{h}_{\mathbb{R}}^{*}$ into open regions, called alcoves. The fundamental alcove $A_{\circ}$ is given by

$$
A_{\circ}:=\left\{\lambda \in \mathfrak{h}_{\mathbb{R}}^{*} \mid 0<\left\langle\lambda, \alpha^{\vee}\right\rangle<1 \text { for all } \alpha \in \Phi^{+}\right\}
$$

\subsection{Weyl groups}

Let $W$ be the Weyl group of the root system $\Phi$ discussed above. The length function on $W$ is denoted by $\ell(\cdot)$. The Bruhat order on $W$ is defined by its covers $w \lessdot w s_{\alpha}$, for $\alpha \in \Phi^{+}$, if $\ell\left(w s_{\alpha}\right)=\ell(w)+1$. Define $w \triangleleft w s_{\alpha}$, for $\alpha \in \Phi^{+}$, if $\ell\left(w s_{\alpha}\right)=\ell(w)-2 \mathrm{ht}\left(\alpha^{\vee}\right)+1$. The quantum Bruhat graph [BFP99] is the directed graph on $W$ with edges labeled by positive roots

$$
w \stackrel{\alpha}{\longrightarrow} w s_{\alpha} \quad \text { for } w \lessdot w s_{\alpha} \text { or } w \triangleleft w s_{\alpha} .
$$

We denote this graph by $\mathrm{QB}(W)$. We will sometimes refer to edges in $\mathrm{QB}(W)$ where $w \lessdot w s_{\alpha}$ as up steps, and edges where $w \triangleleft w s_{\alpha}$ as down steps. See Figure 1 for an example.

We recall an important topological property of $\mathrm{QB}(W)$, called shellability, which was proved in [BFP99]. This is defined with respect to a reflection ordering on the positive roots [D93].

Theorem 2.1. [BFP99] Fix a reflection ordering on $\Phi^{+}$. For any pair of elements $v, w \in W$, there is a unique path from $v$ to $w$ in the quantum Bruhat graph $\mathrm{QB}(W)$ such that its sequence of edge labels is strictly increasing (resp., decreasing) with respect to the reflection ordering.

\subsection{Kirillov-Reshetikhin (KR) crystals}

Given a symmetrizable Kac-Moody algebra $\mathfrak{g}$, a $\mathfrak{g}$-crystal is a non-empty set $B$ together with maps $e_{i}, f_{i}: B \rightarrow B \cup\{\mathbf{0}\}$ for $i \in I$ (where $I$ indexes the simple roots corresponding to $\mathfrak{g}$, as usual, and $\mathbf{0} \notin B$ ), and wt $: B \rightarrow \Lambda$. We require $b^{\prime}=f_{i}(b)$ if and only if $b=e_{i}\left(b^{\prime}\right)$, and wt $\left(f_{i}(b)\right)=\operatorname{wt}(b)-\alpha_{i}$. The maps $e_{i}$ and $f_{i}$ are called crystal operators and are represented as arrows $b \rightarrow b^{\prime}=f_{i}(b)$ colored $i$; thus they endow $B$ with the structure of a colored directed graph. For $b \in B$, we set $\varepsilon_{i}(b):=\max \left\{k \mid e_{i}^{k}(b) \neq \mathbf{0}\right\}$, and $\varphi_{i}(b):=\max \left\{k \mid f_{i}^{k}(b) \neq \mathbf{0}\right\}$. Given two $\mathfrak{g}$-crystals $B_{1}$ and $B_{2}$, we define their tensor product $B_{1} \otimes B_{2}$ as follows. As a set, $B_{1} \otimes B_{2}$ is the Cartesian product of the two sets. For $b=b_{1} \otimes b_{2} \in B_{1} \otimes B_{2}$, the weight function is simply $\mathrm{wt}(b):=\mathrm{wt}\left(b_{1}\right)+\mathrm{wt}\left(b_{2}\right)$. The crystal operators are given by

$$
f_{i}\left(b_{1} \otimes b_{2}\right):= \begin{cases}f_{i}\left(b_{1}\right) \otimes b_{2} & \text { if } \varepsilon_{i}\left(b_{1}\right) \geq \varphi_{i}\left(b_{2}\right) \\ b_{1} \otimes f_{i}\left(b_{2}\right) & \text { otherwise }\end{cases}
$$

and similarly for $e_{i}$. The highest weight crystal $B(\lambda)$ of highest weight $\lambda \in \Lambda^{+}$is a certain crystal with a unique element $u_{\lambda}$ such that $e_{i}\left(u_{\lambda}\right)=\mathbf{0}$ for all $i \in I$ and $\operatorname{wt}\left(u_{\lambda}\right)=\lambda$. It encodes the structure of the crystal basis of the $U_{q}(\mathfrak{g})$-irreducible representation with highest weight $\lambda$ as $q$ goes to 0 .

A Kirillov-Reshetikhin (KR) crystal [KR90] is a finite crystal $B^{r, s}$ for an affine algebra, labeled by a rectangle of height $r$ and width $s$, where $r \in I \backslash\{0\}$ and $s$ is any positive integer. We refer, throughout, to the untwisted affine types $A_{n-1}^{(1)}-G_{2}^{(1)}$, and only consider column shape KR crystals $B^{r, 1}$. 
As an example, consider the KR crystal $B^{r, 1}$ of type $A_{n-1}^{(1)}$ with $r \in\{1,2, \ldots, n-1\}$, for which we have a simple tableau model. As a classical type $A_{n-1}$ crystal, $B^{r, 1}$ is isomorphic to the corresponding crystal $B\left(\omega_{r}\right)$. Recall that an element $b \in B\left(\omega_{r}\right)$ is represented by a strictly increasing filling of a height $r$ column, with entries in $[n]:=\{1, \ldots, n\}$. There is a simple construction of the crystal operators on a tensor product of (column shape) KR crystals of type $A_{n-1}^{(1)}$, which is based on (4).

We refer again to (column shape) KR crystals of arbitrary (untwisted) type. Let $\lambda=\left(\lambda_{1} \geq \lambda_{2} \geq \ldots\right)$ be a partition, and $\lambda^{\prime}$ the conjugate partition. We define $B^{\otimes \lambda}:=\bigotimes_{i=1}^{\lambda_{1}} B^{\lambda_{i}^{\prime}, 1}$. More generally, given a composition $\mathbf{p}=\left(p_{1}, \ldots, p_{k}\right)$, we define $B^{\otimes \mathbf{p}}:=\bigotimes_{i=1}^{k} B^{p_{i}, 1}$. (In both cases, we assume that the corresponding column shape KR crystals exist.) We denote such a tensor product generically by $B$.

Remarks 2.2. (1) It is known that $B$ is connected as an affine crystal, but disconnected as a classical crystal (i.e., with the 0 -arrows removed).

(2) Let $\mathbf{p}^{\prime}$ be a composition obtained from $\mathbf{p}$ by permuting its parts. There is an affine crystal isomorphism between $B^{\otimes \mathbf{p}}$ and $B^{\otimes \mathbf{p}^{\prime}}$, unique by the previous remark, called the combinatorial $R$-matrix.

We need to distinguish certain arrows in $B$, related to affine Demazure crystals, as we shall explain.

Definition 2.3. An arrow $b \rightarrow f_{i}(b)$ in $B$ is called a Demazure arrow if $i \neq 0$, or $i=0$ and $\varepsilon_{0}(b) \geq 1$. An arrow $b \rightarrow f_{i}(b)$ in $B$ is called a dual Demazure arrow if $i \neq 0$, or $i=0$ and $\varphi_{i}(b) \geq 2$.

Remarks 2.4. (1) By Fourier-Littelmann [FL06], in simply-laced types, the tensor product of KR crystals $B$ is isomorphic, as a classical crystal (discard the affine 0-arrows) with a certain Demazure crystal for the corresponding affine algebra. (Demazure modules are submodules of highest weight ones determined by a Borel subalgebra acting on an extremal weight vector.) Moreover, by [FSS07], the 0-arrows in the latter correspond precisely to the Demazure arrows in $B$.

(2) In the case when all of the tensor factors in $B$ are perfect crystals [HK00], $B$ remains connected upon removal of the non-Demazure (resp. non-dual Demazure) 0-arrows.

(3) In the classical types, $B^{k, 1}$ is perfect as follows: in types $A_{n-1}^{(1)}$ and $D_{n}^{(1)}$ for all $k$, in type $B_{n}^{(1)}$ only for $k \neq n$, and in type $C_{n}^{(1)}$ only for $k=n$ (using the standard indexing of the Dynkin diagram); in other words, for all the Dynkin nodes in simply-laced types, and only for the nodes corresponding to the long roots in non-simply-laced types. It was conjectured in $\left[\mathrm{HKO}^{+} 99\right]$ that the same is true in the exceptional types. In type $G_{2}^{(1)}$ this was confirmed in [Yam98], while for types $E_{6,7.8}^{(1)}$ and $F_{4}^{(1)}$ it was checked by computer, based on a model closely related to the quantum alcove model $\left[\mathrm{LNS}^{+} 13 \mathrm{~b}\right]$.

\subsection{The quantum alcove model}

In this section we recall the quantum alcove model, which is a model for KR crystals corresponding to a fixed untwisted affine Lie algebra $\widehat{\mathfrak{g}}$. This model is based on the combinatorics of the root system of the corresponding finite-dimensional Lie algebra $\mathfrak{g}$, so we use freely the notation in Section 2.1 .

We say that two alcoves are adjacent if they are distinct and have a common wall. Given a pair of adjacent alcoves $A$ and $B$, we write $A \stackrel{\beta}{\longrightarrow} B$ if the common wall is of the form $H_{\beta, k}$ and the root $\beta \in \Phi$ points in the direction from $A$ to $B$.

Definition 2.5. An alcove path is a sequence of alcoves $\left(A_{0}, A_{1}, \ldots, A_{m}\right)$ such that $A_{j-1}$ and $A_{j}$ are adjacent, for $j=1, \ldots, m$. We say that an alcove path is reduced if it has minimal length among all alcove paths from $A_{0}$ to $A_{m}$.

Let $A_{\lambda}=A_{\circ}+\lambda$ be the translation of the fundamental alcove $A_{\circ}$ by the weight $\lambda$. 
Definition 2.6. The sequence of roots $\left(\beta_{1}, \beta_{2}, \ldots, \beta_{m}\right)$ is called a $\lambda$-chain if

$$
A_{0}=A_{\circ} \stackrel{-\beta_{1}}{\longrightarrow} A_{1} \stackrel{-\beta_{2}}{\longrightarrow} \cdots \stackrel{-\beta_{m}}{\longrightarrow} A_{m}=A_{-\lambda}
$$

is a reduced alcove path.

We now fix a dominant weight $\lambda$ and an alcove path $\Pi=\left(A_{0}, \ldots, A_{m}\right)$ from $A_{0}=A_{\circ}$ to $A_{m}=$ $A_{-\lambda}$. Note that $\Pi$ is determined by the corresponding $\lambda$-chain $\Gamma:=\left(\beta_{1}, \ldots, \beta_{m}\right)$, which consists of positive roots. A specific choice of a $\lambda$-chain, called a lex $\lambda$-chain and denoted $\Gamma_{\text {lex }}$, is given in [LP08, Proposition 4.2]; this choice depends on a total order on the simple roots.

Given the $\lambda$-chain $\Gamma$ above, we let $r_{i}:=s_{\beta_{i}}$, and let $\widehat{r_{i}}$ be the affine reflection in the hyperplane containing the common face of $A_{i-1}$ and $A_{i}$, for $i=1, \ldots, m$; so $\widehat{r}_{i}:=s_{\beta_{i},-l_{i}}$, where $l_{i}:=\left|\left\{j<i ; \beta_{j}=\beta_{i}\right\}\right|$. We define $\widetilde{l}_{i}:=\left\langle\lambda, \beta_{i}^{\vee}\right\rangle-l_{i}=\left|\left\{j \geq i ; \beta_{j}=\beta_{i}\right\}\right|$. Let $J=\left\{j_{1}<j_{2}<\cdots<j_{s}\right\} \subseteq[m]$, whose elements are called folding positions. We say that $j_{i}$ is a positive folding position if $r_{j_{1}} r_{j_{2}} \ldots r_{j_{i-1}}<$ $r_{j_{1}} r_{j_{2}} \ldots r_{j_{i}}$, and a negative folding position otherwise. We denote the positive folding positions by $J^{+}$, and the negative ones by $J^{-}$. We define the weight $\mu(J)$ and the height statistic by

$$
\mu=\mu(J):=-\widehat{r}_{j_{1}} \widehat{r}_{j_{2}} \ldots \widehat{r}_{j_{s}}(-\lambda), \quad \operatorname{height}(J):=\sum_{j \in J^{-}} \widetilde{l}_{j}
$$

Definition 2.7. A subset $J=\left\{j_{1}<j_{2}<\cdots<j_{s}\right\} \subseteq[m]$ (possibly empty) is admissible if we have the following path in $\mathrm{QB}(W)$ :

$$
1 \stackrel{\beta_{j_{1}}}{\longrightarrow} r_{j_{1}} \stackrel{\beta_{j_{2}}}{\longrightarrow} r_{j_{1}} r_{j_{2}} \stackrel{\beta_{j_{3}}}{\longrightarrow} \cdots \stackrel{\beta_{j_{s}}}{\longrightarrow} r_{j_{1}} r_{j_{2}} \cdots r_{j_{s}}
$$

We let $\mathcal{A}(\Gamma)$ be the collection of admissible subsets corresponding to the $\lambda$-chain $\Gamma$. When $\Gamma$ is clear from the context, we may use the notation $\mathcal{A}(\lambda)$ instead.

Remark 2.8. If we restrict to admissible subsets for which the path (7) has no down steps, we recover the classical alcove model in [LP07, LP08].

Combinatorial crystal operators $f_{i}$ and $e_{i}$ (where $i$ indexes the simple roots corresponding to $\widehat{\mathfrak{g}}$ ) were constructed on $\mathcal{A}(\Gamma)$ and their properties were studied in [LL14]. In particular, the crystal operator $f_{i}$ applied to an admissible subset $J$ adds a folding position to $J$, while at the same time possibly removing a folding position from $J$.

We summarize the results in $\left[\mathrm{LNS}^{+} 13 \mathrm{~b}, \mathrm{LNS}^{+} 12\right]$ about the applications of the quantum alcove model.

Theorem 2.9. $\left[\mathrm{LNS}^{+} 13 \mathrm{~b}\right]$ Consider a composition $\mathbf{p}=\left(p_{1}, \ldots, p_{k}\right)$ and the corresponding KR crystal $B:=\bigotimes_{i=1}^{k} B^{p_{i}, 1}$. Let $\lambda:=\omega_{p_{1}}+\ldots+\omega_{p_{k}}$, and let $\Gamma_{\text {lex }}$ be a corresponding lex $\lambda$-chain.

(1) The (combinatorial) crystal $\mathcal{A}\left(\Gamma_{\text {lex }}\right)$ is isomorphic to the subgraph of $B$ consisting of the dual Demazure arrows, via a specific bijection which preserves the weights of the vertices.

(2) If the vertex b of $B$ corresponds to $J$ under the isomorphism in part (1), then the energy at b is given, up to a global constant, by - height $(J)$.

Remark 2.10. The isomorphism in Theorem 2.9 (1) is canonical, so we identify the two crystals. 


\subsection{Specializing the quantum alcove model to type $A$}

We start with the basic facts about the root system of type $A_{n-1}$. We can identify the space $\mathfrak{h}_{\mathbb{R}}^{*}$ with the quotient $V:=\mathbb{R}^{n} / \mathbb{R}(1, \ldots, 1)$, where $\mathbb{R}(1, \ldots, 1)$ denotes the subspace in $\mathbb{R}^{n}$ spanned by the vector $(1, \ldots, 1)$. Let $\varepsilon_{1}, \ldots, \varepsilon_{n} \in V$ be the images of the coordinate vectors in $\mathbb{R}^{n}$. The root system is $\Phi=$ $\left\{\alpha_{i j}:=\varepsilon_{i}-\varepsilon_{j}: i \neq j, 1 \leq i, j \leq n\right\}$. The simple roots are $\alpha_{i}=\alpha_{i, i+1}$, for $i=1, \ldots, n-1$. The weight lattice is $\Lambda=\mathbb{Z}^{n} / \mathbb{Z}(1, \ldots, 1)$. The fundamental weights are $\omega_{i}=\varepsilon_{1}+\ldots+\varepsilon_{i}$, for $i=1, \ldots, n-1$. A dominant weight $\lambda=\lambda_{1} \varepsilon_{1}+\ldots+\lambda_{n-1} \varepsilon_{n-1}$ is identified with the partition $\left(\lambda_{1} \geq \lambda_{2} \geq \ldots \geq \lambda_{n-1} \geq\right.$ $\lambda_{n}=0$ ) having at most $n-1$ parts. Considering the Young diagram of the dominant weight $\lambda$ as a concatenation of columns, whose heights are $\lambda_{1}^{\prime}, \lambda_{2}^{\prime}, \ldots$, corresponds to expressing $\lambda$ as $\omega_{\lambda_{1}^{\prime}}+\omega_{\lambda_{2}^{\prime}}+\ldots$ (as usual, $\lambda^{\prime}$ is the conjugate partition to $\lambda$ ).

The Weyl group $W$ is the symmetric group $S_{n}$, which acts on $V$ by permuting the coordinates $\varepsilon_{1}, \ldots, \varepsilon_{n}$. Permutations $w \in S_{n}$ are written in one-line notation $w=w(1) \ldots w(n)$. For simplicity, we use the same notation $(i, j)$ with $1 \leq i<j \leq n$ for the root $\alpha_{i j}$ and the reflection $s_{\alpha_{i j}}$, which is the transposition $t_{i j}$.

We now consider the specialization of the alcove model to type $A$. For any $k=1, \ldots, n-1$, we have the following $\omega_{k}$-chain, from $A_{\circ}$ to $A_{-\omega_{k}}$, denoted by $\Gamma(k)$ :

$$
\begin{array}{llll}
((k, k+1), & (k, k+2) & , \ldots, & (k, n), \\
(k-1, k+1), & (k-1, k+2) & , \ldots, & (k-1, n), \\
\vdots & \vdots & & \vdots \\
(1, k+1), & (1, k+2) & , \ldots, & (1, n)) .
\end{array}
$$

Fix a dominant weight $\lambda$, for which we use the partition notation above. We construct a $\lambda$-chain $\Gamma=\left(\beta_{1}, \beta_{2}, \ldots, \beta_{m}\right)$ as the concatenation $\Gamma:=\Gamma^{1} \ldots \Gamma^{\lambda_{1}}$, where $\Gamma^{j}=\Gamma\left(\lambda_{j}^{\prime}\right)$. Let $J=\left\{j_{1}<\cdots<j_{s}\right\}$ be a set of folding positions in $\Gamma$, not necessarily admissible, and let $T$ be the corresponding list of roots of $\Gamma$, also viewed as transpositions. The factorization of $\Gamma$ induces a factorization on $T$ as $T=T^{1} T^{2} \ldots T^{\lambda_{1}}$. We denote by $T^{1} \ldots T^{j}$ the permutation obtained by composing the transpositions in $T^{1}, \ldots, T^{j}$ from left to right. For $w \in W$, written $w=w_{1} w_{2} \ldots w_{n}$, let $w[i, j]=w_{i} \ldots w_{j}$.

Definition 2.11. Let $\pi_{j}=\pi_{j}(T):=T^{1} \ldots T^{j}$. We define the filling map, which associates with each $J \subseteq[m]$ a filling of the Young diagram $\lambda$, by fill $(J)=\operatorname{fill}(T):=C_{1} \ldots C_{\lambda_{1}}$, where $C_{i}:=\pi_{i}\left[1, \lambda_{i}^{\prime}\right]$. In other words, the columns $C_{i}$ of the Young diagram are filled top to bottom by the first $\lambda_{i}^{\prime}$ entries of the permutation $\pi_{i}$. We define the sorted filling map $\operatorname{sfill}(J)$ by sorting ascendingly the columns of fill $(J)$.

Theorem 2.12. [Len12, LL14] The map sfill is the unique affine crystal isomorphism between $\mathcal{A}(\Gamma)$ and the subgraph of $B^{\otimes \lambda}$ consisting of the dual Demazure arrows. In other words, given $\operatorname{sill}(J)=b$, there is a dual Demazure arrow $b \rightarrow f_{i}(b)$ if and only if $f_{i}(J) \neq 0$, and we have $f_{i}(b)=\operatorname{sill}\left(f_{i}(J)\right)$. The map sfill also preserves weights, and translates the height statistic into the Lascoux-Schützenberger charge statistic on fillings.

There is a similar result in type $C$ [Len12], [LS13], [LL14, Section 4.2].

\section{The main results}

In this section we realize the combinatorial $R$-matrix in terms of the quantum alcove model, and show that this model is independent of the choice of a $\lambda$-chain. We start with a preview of the main result. Let 
$\mathbf{p}$ be the composition $\mathbf{p}=\left(p_{1}, \ldots, p_{k}\right)$, and let $\mathbf{p}^{\prime}=\left(p_{1}^{\prime}, \ldots, p_{k}^{\prime}\right)$ be a permutation of $\mathbf{p}$. Let

$$
\begin{aligned}
& B^{\otimes \mathbf{p}}:=B^{p_{1}, 1} \otimes \cdots \otimes B^{p_{k}, 1}, \quad B^{\otimes \mathbf{p}^{\prime}}:=B^{p_{1}^{\prime}, 1} \otimes \cdots \otimes B^{p_{k}^{\prime}, 1} \\
& \Gamma:=\Gamma\left(p_{1}\right) \cdots \Gamma\left(p_{k}\right), \quad \Gamma^{\prime}:=\Gamma\left(p_{1}^{\prime}\right) \cdots \Gamma\left(p_{2}^{\prime}\right),
\end{aligned}
$$

where $\Gamma(i)$ is an $\omega_{i}$-chain; thus, $\Gamma$ and $\Gamma^{\prime}$ are $\lambda$-chains, where $\lambda:=\omega_{p_{1}}+\cdots+\omega_{p_{k}}$. We will show that $\mathcal{A}(\Gamma)$ and $\mathcal{A}\left(\Gamma^{\prime}\right)$ are models for the isomorphic affine crystals $B^{\otimes \mathbf{p}}$ and $B^{\otimes \mathbf{p}^{\prime}}$. Thus, we want to realize the combinatorial $R$-matrix as an affine crystal isomorphism between $\mathcal{A}(\Gamma)$ and $\mathcal{A}\left(\Gamma^{\prime}\right)$.

Example 3.1. Consider type $A_{2}$. Let $\mathbf{p}=(1,2,2,1), \mathbf{p}^{\prime}=(1,2,1,2)$, so $\lambda=(4,2,0)$. Then

$$
B^{\otimes \mathbf{p}}=B^{1,1} \otimes B^{2,1} \otimes B^{2,1} \otimes B^{1,1} \simeq B^{1,1} \otimes B^{2,1} \otimes B^{1,1} \otimes B^{2,1}=B^{\otimes \mathbf{p}^{\prime}} .
$$

We first note that in type $A$ the combinatorial $R$-matrix can be realized by Schützenberger's jeu de taquin (sliding algorithm) on the last two columns, see [Ful97]. For example:

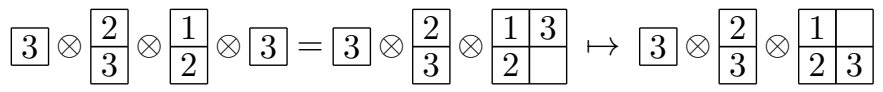

$$
\begin{aligned}
& \mapsto 3 \otimes \frac{2}{3} \otimes \frac{1}{2}=3 \otimes \frac{2}{3} \otimes 2 \otimes 2 \otimes \frac{1}{3} .
\end{aligned}
$$

We now demonstrate how to realize the combinatorial $R$-matrix in the quantum alcove model. Let $\Gamma$ and $\Gamma^{\prime}$ be the $\lambda$-chains corresponding to $\mathbf{p}$ and $\mathbf{p}^{\prime}$ (the bars indicate the splittings into $\omega_{i}$-chains, cf. (9)):

$$
\begin{aligned}
& \Gamma=(\underline{(1,2)}, \underline{(1,3)}|\underline{(2,3)},(1,3)|(2,3), \underline{(1,3)} \mid \underline{(1,2)}, \underline{(1,3)}), \\
& \Gamma^{\prime}=(\underline{(1,2)}, \underline{(1,3)}|\underline{(2,3)},(1,3)| \underline{(1,2)},(1,3) \mid \underline{(2,3)}, \underline{(1,3)}) .
\end{aligned}
$$

In (10) we also showed (via the underlined positions) two admissible subsets: $J=\{1,2,3,6,7,8\}$ in $\mathcal{A}(\Gamma)$ and $J^{\prime}=\{1,2,3,5,7,8\}$ in $\mathcal{A}\left(\Gamma^{\prime}\right)$. Here $J$ is admissible as it corresponds to the path in $\mathrm{QB}(W)$ shown in Figure 1a which starts at the identity and consists of the edges labeled 1,2,3,2,1,2. The details of our construction will be given in Section 3.1 (see Example 3.3; for now, note that $J$ will correspond to $J^{\prime}$ via our construction. We recover the construction above in terms of jeu de taquin since

$$
\operatorname{sfill}(J)=3 \otimes \frac{2}{3} \otimes \frac{1}{2} \otimes 3 \mapsto 3 \otimes \frac{2}{3} \otimes 2 \otimes \frac{1}{3}=\operatorname{sfill}\left(J^{\prime}\right) .
$$

We construct a bijection between $\mathcal{A}(\Gamma)$ and $\mathcal{A}\left(\Gamma^{\prime}\right)$ by generalizing the construction in [Len07], which gives the bijection in the classical case, where admissible subsets correspond to saturated chains in Bruhat order. The bijection in [Len07] is based on a sequence of operations called Yang-Baxter moves.

\subsection{Quantum Yang-Baxter moves}

This section contains our main constructions. We use freely the notation related to the quantum alcove model in Section 2.4. We consider segments of the $\lambda$-chain corresponding to Weyl subgroups of rank 2.

We start by recalling that there are only two reflection orderings on the positive roots corresponding to a dihedral Weyl group of order $2 q$, that is, a Weyl group of type $A_{1} \times A_{1}, A_{2}, C_{2}$, or $G_{2}$ (with $q=2,3,4,6$, 
respectively). Let $\bar{\Phi}$ be the corresponding root system with simple roots $\alpha, \beta$. The two reflection orderings on $\bar{\Phi}^{+}$are given by the following sequence and its reverse:

$$
\beta_{1}:=\alpha, \beta_{2}:=s_{\alpha}(\beta), \quad \beta_{3}:=s_{\alpha} s_{\beta}(\alpha), \ldots, \quad \beta_{q-1}:=s_{\beta}(\alpha), \quad \beta_{q}:=\beta .
$$

Fix $\lambda \in \Lambda^{+}$. Consider an index set $I:=\{\overline{1}<\ldots<\bar{t}<1<\ldots<q<\overline{t+1}<\ldots<\bar{n}\}$. Let $\Gamma=\left\{\beta_{i}\right\}_{i \in I}$ be a $\lambda$-chain, denote $r_{i}:=s_{\beta_{i}}$ as before, and define the sequence of roots $\Gamma^{\prime}=\left\{\beta_{i}^{\prime}\right\}_{i \in I}$ by

$$
\beta_{i}^{\prime}= \begin{cases}\beta_{q+1-i} & \text { if } i \in[q] \\ \beta_{i} & \text { if } i \in I \backslash[q] .\end{cases}
$$

In other words, the sequence $\Gamma^{\prime}$ is obtained from the $\lambda$-chain $\Gamma$ by reversing a certain segment. Now assume that $\left\{\beta_{1}, \ldots, \beta_{q}\right\}$ are the positive roots (without repetition) of a rank two root subsystem $\bar{\Phi}$ of $\Phi$. The corresponding dihedral reflection group $\bar{W}$ is a subgroup of the Weyl group $W$.

Proposition 3.2. [Len07]

(1) The sequence $\Gamma^{\prime}$ is also a $\lambda$-chain, and the sequence $\left(\beta_{1}, \ldots, \beta_{q}\right)$ is a reflection ordering of $\bar{\Phi}^{+}$.

(2) We can obtain any $\lambda$-chain from any other $\lambda$-chain by moves of the form $\Gamma \rightarrow \Gamma^{\prime}$.

Let us now map the admissible subsets in $\mathcal{A}(\Gamma)$ to those in $\mathcal{A}\left(\Gamma^{\prime}\right)$. To this end, fix a reflection ordering of $\Phi^{+}$compatible with the above ordering $\left(\beta_{1}, \ldots, \beta_{q}\right)$ of $\bar{\Phi}$; this clearly exists (take any reflection ordering of $\Phi^{+}$, and reverse it if needed). Now fix an admissible subset $J=\left\{j_{1}<\cdots<j_{s}\right\}$ in $\mathcal{A}(\Gamma)$. Define $w(J):=r_{j_{1}} r_{j_{2}} \cdots r_{j_{s}}$, and

$$
u:=w(J \cap\{\overline{1}, \ldots, \bar{t}\}), \quad \text { and } \quad w:=w(J \cap(\{\overline{1}, \ldots, \bar{t}\} \cup[q])) .
$$

Note that, by the definition of $\mathcal{A}(\Gamma)$, we have a path in $\mathrm{QB}(W)$ from $u$ to $w$ with increasing edge labels $J \cap[q]$ (here we identify an edge label $\beta_{i}$ with $i$, for $i \in[q]$ ). By the shellability property of $\mathrm{QB}(W)$, that is, by Theorem 2.1. there is another path in $\mathrm{QB}(W)$ from $u$ to $w$ whose edge labels (in $\Phi^{+}$) increase with respect to the reverse of the reflection ordering considered above. In fact, by the proof of Theorem 2.1 in [BFP99], these edge labels are also in $\bar{\Phi}^{+}$, since the edge labels of the first path had this property. Thus, by now identifying the label $\beta_{i}^{\prime}$ with $i$, we can view the edge labels of the new path as a subset of $[q]$, which we denote by $Y_{u, w}(J \cap[q])$.

It is clear that we have a bijection $Y: \mathcal{A}(\Gamma) \rightarrow \mathcal{A}\left(\Gamma^{\prime}\right)$ given by

$$
Y(J):=(J \backslash[q]) \cup Y_{u, w}(J \cap[q]) .
$$

We call the moves $J \mapsto Y(J)$ quantum Yang-Baxter moves. They generalize the Yang-Baxter moves in [Len07], which correspond to saturated chains in the Bruhat order (i.e., there are no down steps).

Example 3.3. We continue Example 3.1. In (10) we obtain $\Gamma^{\prime}$ from $\Gamma$ by reversing the first three roots in the right half of $\Gamma$. We will use the conventions of this section and (re)label this segment of $\Gamma$ by $\left(\beta_{1}, \beta_{2}, \beta_{3}\right)$. We also let $s_{1}=s_{\beta_{1}}$ and $s_{2}=s_{\beta_{3}}$. We have $u=s_{1} s_{2} s_{1}=321$, and $w=s_{2}=213$, in one line notation. In this case, we have $Y_{u, w}(\{2,3\})=\{1,3\}$, see Figure 1a Hence $Y(\{1,2,3,6,7,8\})=$ $\{1,2,3,5,7,8\}$, where now the indexing corresponds to the entire $\lambda$-chain, cf. Example 3.1 
We now describe the quantum Yang-Baxter moves in types $A_{2}, C_{2}$, and $B_{2}$; type $G_{2}$ is more involved, but still manageable. Our description is in terms of the corresponding dihedral reflection group $\bar{W}$, which is a subgroup of the Weyl group $W$ above. The following result is based on [BFP99, Lemma 5.1].

Proposition 3.4. Given $w \in W$, let $\lfloor w\rfloor$ be the minimum length representative of the coset $w \bar{W}$, and write $w=\lfloor w\rfloor \bar{w}$, where $\bar{w} \in \bar{W}$. Under the bijection $\bar{w} \mapsto\lfloor w\rfloor \bar{w}$ between $\bar{W}$ and $\bar{w}$, every edge of the graph on $w \bar{W}$ induced from $\mathrm{QB}(W)$ corresponds to an edge of $\mathrm{QB}(\bar{W})$.

By Proposition 3.4, the map $Y_{u, w}$ used to define the quantum Yang-Baxter moves in (14) depends only on $\bar{u}$ and $\bar{w}$, so we will denote it by $Y_{\bar{u}}, \bar{w}$. Hence it suffices to focus on the quantum Bruhat graphs for the dihedral Weyl groups. The ones of type $A_{2}$ and $C_{2}$ are shown in Figure 1, where the edge labels correspond to the reflection ordering (11), and $s_{1}:=s_{\beta_{1}}, s_{2}:=s_{\beta_{q}}$. The graph of type $B_{2}$ is identical with the one of type $C_{2}$ if we set $\beta_{1}=\varepsilon_{2}$ (short root, like in type $C_{2}$ ), $\beta_{4}=\varepsilon_{1}-\varepsilon_{2}$ (long root), and $s_{1}:=s_{\beta_{1}}, s_{2}:=s_{\beta_{4}}$, as above.

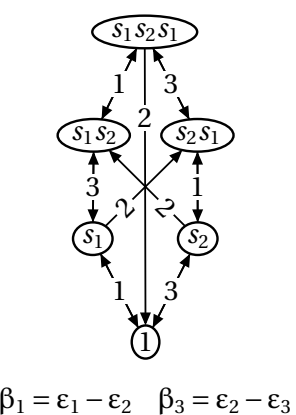

(a) Type $A_{2}$

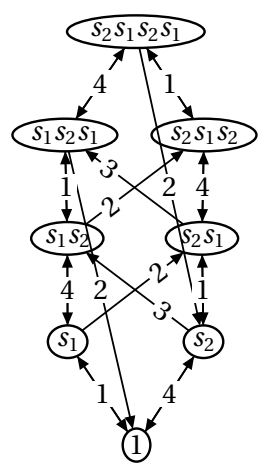

$\beta_{1}=\varepsilon_{1}-\varepsilon_{2}, \quad \beta_{4}=2 \varepsilon_{2}$

(b) Type $C_{2}$

Fig. 1: Quantum Bruhat graphs.

Let us now recall from [Len07] the explicit description of the classical Yang-Baxter moves, i.e., of the map $J \rightarrow Y_{\bar{u}, \bar{w}}(J)$ on subsets of $[q]$, for $\bar{u}<\bar{w}$ in $\bar{W}$. Recall that $J$ and $Y_{\bar{u}, \bar{w}}(J)$ correspond to saturated chains in Bruhat order from $\bar{u}$ to $\bar{w}$, whose edge labels increase, resp. decrease, with respect to the reflection ordering (11); in fact, as explained in Section 3.1, an index $i$ in $J$ corresponds to the root/edge label $\beta_{i}$, whereas an index $i$ in $Y_{\bar{u}, \bar{w}}(J)$ corresponds to the root $\beta_{i}^{\prime}:=\beta_{q+1-i}$, cf. (12). The classical moves can be described in a uniform way (i.e., for all types), and only in terms of $a:=\ell(\bar{u})$ and $b:=\ell(\bar{w})$, as shown in Figure 2 The non-classical quantum Yang-Baxter moves $J \rightarrow Y_{\bar{u}}, \bar{w}(J)$ do not have a uniform description and are listed in Figure 3 , by the corresponding starting vertex $\bar{u}$. The top and bottom rows are the sequences $J$ and $Y_{\bar{u}, \bar{w}}(J)$, respectively, while we also have to consider the reverse moves $Y_{\bar{u}, \bar{w}}(J) \rightarrow J$; the mentioned sequences correspond to paths in $\mathrm{QB}(\bar{W})$, as explained in Section 3.1 (see also the convention related to the edge labels, which was recalled above, in connection to the classical moves). The symbol $*$ is used to indicate the down edges in $\mathrm{QB}(\bar{W})$. 
Case 0: $\emptyset \leftrightarrow \emptyset$ if $a=b$.

Case 1.1: $\{1\} \leftrightarrow\{q\}$ if $0 \leq a=b-1 \leq q-1$.

Case 1.2: $\{q-a\} \leftrightarrow\{a+1\}$ if $0<a=b-1<q-1$.

Case 2.1: $\{1, a+2, a+3, \ldots, b\} \leftrightarrow\{a+1, a+2, \ldots, b-1, q\}$ if $0 \leq a<a+2 \leq b<q$.

Case 2.2: $\{1, a+2, a+3, \ldots, b-1, q\} \leftrightarrow\{a+1, a+2, \ldots, b\}$ if $0<a<a+2 \leq b \leq q$.

Case 3: $\quad[q] \leftrightarrow[q]$ if $a=0$ and $b=q$.

Fig. 2: The classical Yang-Baxter moves.

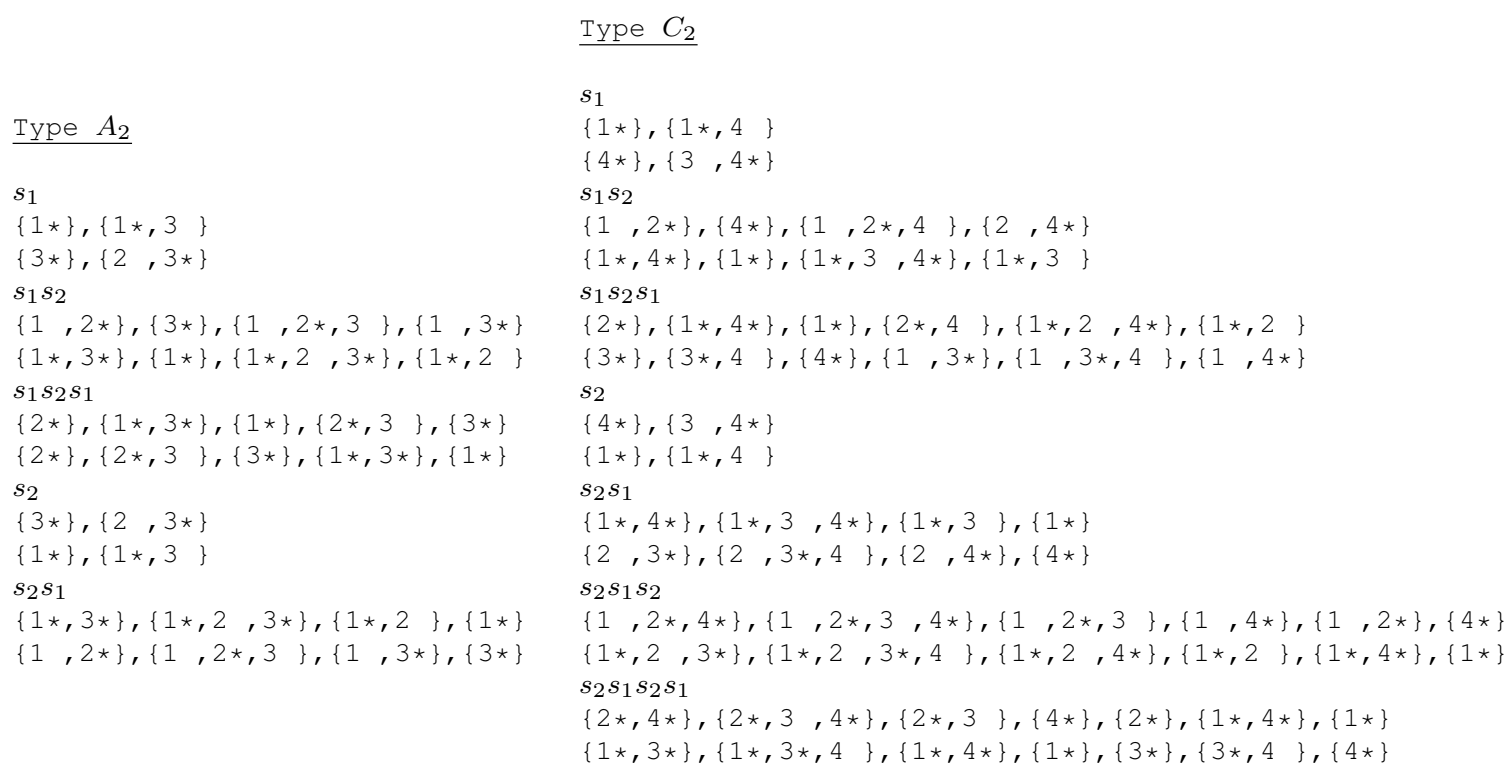

Fig. 3: The non-classical Yang-Baxter moves for types $A_{2}$ and $C_{2}$.

\subsection{Properties of the quantum Yang-Baxter moves}

Using the same notation as in Section 3.1, we present the main properties of the quantum Yang-Baxter moves, concerning the quantities they preserve, and their interaction with the crystal operators.

Proposition 3.5. The quantum Yang-Baxter moves preserve the Weyl group element $w(\cdot)$, as well as the weight $\mu(\cdot)$ and the height statistic (defined in (6)); in other words, we have

$$
w(J)=w(Y(J)), \quad \mu(J)=\mu(Y(J)), \quad \operatorname{height}(J)=\operatorname{height}(Y(J)),
$$

where the left hand sides are computed with respect to $\Gamma$, and the right hand sides with respect to $\Gamma^{\prime}$.

Theorem 3.6 below generalizes the similar result in [Len07] for the classical Yang-Baxter moves.

Theorem 3.6. The crystal operators commute with the quantum Yang-Baxter moves, that is, $f_{i}$ is defined on an admissible subset $J$ if and only if it is defined on $Y(J)$, and we have $Y\left(f_{i}(J)\right)=f_{i}(Y(J))$. 


\subsection{Corollaries and conjectures}

In this section we state some corollaries of the results in the previous section which are, in fact, the main results of the paper. We also discuss possible strengthenings of these results.

Theorem 3.6 and Proposition 3.5 immediately imply the following corollary (cf. also Proposition 3.2, which essentially says that the quantum alcove model is independent of the choice of a $\lambda$-chain.

Corollary 3.7. Given $\lambda$-chains $\Gamma$ and $\Gamma^{\prime}$, there is an affine crystal isomorphism between $\mathcal{A}(\Gamma)$ and $\mathcal{A}\left(\Gamma^{\prime}\right)$, realized by composing quantum Yang-Baxter moves; it preserves the weights and heights of the vertices.

By composing the explicit bijection between $B^{\otimes \mathbf{p}}$ and $\mathcal{A}\left(\Gamma_{\text {lex }}\right)$ in Theorem 2.9 with an affine crystal isomorphism between $\mathcal{A}\left(\Gamma_{\text {lex }}\right)$ and $\mathcal{A}(\Gamma)$ realized by quantum Yang-Baxter moves (where $\Gamma$ is an arbitrary $\lambda$-chain, see Corollary 3.7, we obtain the following strengthening of Theorem 2.9

Corollary 3.8. Theorem 2.9 holds for any choice of a $\lambda$-chain, based on the bijection mentioned above.

Remark 3.9. There are several ways to connect two $\lambda$-chains $\Gamma$ to $\Gamma^{\prime}$ via the moves in Proposition 3.2 . A priori, the corresponding compositions of quantum Yang-Baxter moves give different affine crystal isomorphisms between $\mathcal{A}(\Gamma)$ and $\mathcal{A}\left(\Gamma^{\prime}\right)$ in Corollary 3.7 Therefore, in Corollary 3.8 we have a collection of a priori different affine crystal isomorphisms between $B^{\otimes \mathbf{p}}$ and $\mathcal{A}(\Gamma)$, for a fixed $\lambda$-chain $\Gamma$. All this is due to the fact that $B^{\otimes \mathbf{p}}$ is not necessarily connected under the dual Demazure arrows, cf. Remark 2.2 (1) and Remark 2.4 (2). However, we make the following conjecture.

Conjecture 3.10. All the affine crystal isomorphisms between $\mathcal{A}(\Gamma)$ and $\mathcal{A}\left(\Gamma^{\prime}\right)$ in Corollary 3.7 are identical. The same is true about the isomorphisms between $B^{\otimes \mathbf{p}}$ and $\mathcal{A}(\Gamma)$ in Corollary 3.8

Remark 3.11. If all the tensor factors in $B^{\otimes \mathbf{p}}$ are perfect crystals, then there is a unique affine crystal isomorphism between $B^{\otimes \mathbf{p}}$ and $\mathcal{A}(\Gamma)$, by Remark 2.4 (2). Conjecture 3.10 then follows.

Now let $\mathbf{p}$ be a composition and $\mathbf{p}^{\prime}$ a permutation of it. Recall the corresponding $\lambda$-chains $\Gamma$ and $\Gamma^{\prime}$ constructed in (9). By Corollary 3.8, we have affine crystal isomorphisms between $B^{\otimes \mathbf{p}}$ and $\mathcal{A}(\Gamma)$, as well as between $B^{\otimes \mathbf{p}}$ and $\mathcal{A}\left(\Gamma^{\prime}\right)$. Also recall from Remark 2.2 (2) the combinatorial $R$-matrix $B^{\otimes \mathbf{p}} \cong B^{\otimes \mathbf{p}^{\prime}}$. The uniqueness property in Remark 3.11 leads to the following result.

Corollary 3.12. Suppose that all the tensor factors in $B^{\otimes \mathbf{p}}$ are perfect crystals. Then the quantum YangBaxter moves realize the combinatorial R-matrix as an affine crystal isomorphism between $\mathcal{A}(\Gamma)$ and $\mathcal{A}\left(\Gamma^{\prime}\right)$, in the sense mentioned above.

Remark 3.13. For the general case of Corollary 3.12, we need to realize the non-dual Demazure 0 -arrows in the quantum alcove model, cf. Theorem 2.9 and generalize Theorem 3.6 accordingly.

\section{References}

[BFP99] F. Brenti, S. Fomin, and A. Postnikov. Mixed Bruhat operators and Yang-Baxter equations for Weyl groups. Int. Math. Res. Not., 8:419-441, 1999.

[D93] M. Dyer. Hecke algebras and shellings of Bruhat intervals. Compositio Math., 89:91-115, 1993.

[FL06] G. Fourier and P. Littelmann. Tensor product structure of affine Demazure modules and limit constructions. Nagoya Math. J., 182:171-198, 2006.

[FOS09] G. Fourier, M. Okado, and A. Schilling. Kirillov-Reshetikhin crystals for nonexceptional types. Adv. Math., 222:1080-1116, 2009. 
[FSS07] G. Fourier, A. Schilling, and M. Shimozono. Demazure structure inside Kirillov-Reshetikhin crystals. J. Algebra, 309:386-404, 2007.

[Fu197] W. Fulton. Young Tableaux. Cambridge University Press, 1997.

$\left[\mathrm{HKO}^{+} 99\right]$ G. Hatayama, A. Kuniba, M. Okado, T. Takagi, and Y. Yamada. Remarks on fermionic formula. In Recent developments in quantum affine algebras and related topics (Raleigh, NC, 1998), volume 248 of Contemp. Math., pages 243-291. Amer. Math. Soc., Providence, RI, 1999.

[HK00] J. Hong and S.J. Kang. Introduction to Quantum Groups and Crystal Bases, volume 42 of Graduate Studies in Mathematics. Amer. Math. Soc., 2000.

[Kas91] M. Kashiwara. On crystal bases of the $q$-analogue of universal enveloping algebras. Duke Math. J., 63:465-516, 1991.

[KR90] A. Kirillov and N. Reshetikhin. Representations of Yangians and multiplicities of the inclusion of the irreducible components of the tensor product of representations of simple Lie algebras. J. Sov. Math., 52:3156-3164, 1990.

[Lec02] C. Lecouvey. Schensted-type correspondence, plactic monoid, and jeu de taquin for type $C_{n}$. J. Algebra, 247:295-331, 2002.

[Lec03] C. Lecouvey. Schensted-type correspondences and plactic monoids for types $B_{n}$ and $D_{n}$. J. Algebraic Combin., 18:99-133, 2003.

[Len07] C. Lenart. On the combinatorics of crystal graphs, I. Lusztig's involution. Adv. Math., 211:324-340, 2007.

[Len12] C. Lenart. From Macdonald polynomials to a charge statistic beyond type A. J. Combin. Theory Ser. A, 119:683-712, 2012.

[LL14] C. Lenart and A. Lubovsky. A generalization of the alcove model and its applications. J. Algebraic Combin., 41:751-783, 2015.

[LL15] C. Lenart and A. Lubovsky. A uniform realization of the combinatorial $R$-matrix. arXiv:1503.01765

[LNS $\left.{ }^{+} 12\right]$ C. Lenart, S. Naito, D. Sagaki, A. Schilling, and M. Shimozono. A uniform model for KirillovReshetikhin crystals I: Lifting the parabolic quantum Bruhat graph. Int. Math. Res. Not., 7:1848-1901, 2015.

$\left[\mathrm{LNS}^{+}\right.$13b] C. Lenart, S. Naito, D. Sagaki, A. Schilling, and M. Shimozono. A uniform model for KirillovReshetikhin crystals II: Path models and $P=X$. arXiv:1402.2203.

[LP07] C. Lenart and A. Postnikov. Affine Weyl groups in K-theory and representation theory. Int. Math. Res. Not., pages 1-65, 2007. Art. ID rnm038.

[LP08] C. Lenart and A. Postnikov. A combinatorial model for crystals of Kac-Moody algebras. Trans. Amer. Math. Soc., 360:4349-4381, 2008.

[LS13] C. Lenart and A. Schilling. Crystal energy via the charge in types $A$ and C. Math. Z., 273:401-426, 2013.

[Lit95] P. Littelmann. Paths and root operators in representation theory. Ann. of Math. (2), 142:499-525, 1995.

[Yam98] Y. Yamane. Perfect crystals of $U_{q}\left(G_{2}^{(1)}\right)$. J. Algebra, 210:440-486, 1998. 